\title{
AVALIAÇÕES MUNICIPAIS: UMA REVISÃO BIBLIOGRÁFICA (1990-2016)
}

http://dx.doi.org/10.5902/2318133836817

\author{
Antônia Bruna da Silva ${ }^{1}$ \\ Maria Beatriz Fernandes²
}

\begin{abstract}
Resumo
Neste texto apresentam-se resultados de pesquisa que foi desenvolvida com o objetivo de localizar e analisar a produção científica sobre iniciativas municipais de avaliação veiculada em cinco fontes especializadas em avaliação educacional, no período de 1990 a 2016, com vistas destacar os principais aspectos característicos dessas avaliações. Nas bases de dados pesquisadas identificou-se um total de dezoito publicações que contêm em seus títulos algum indicativo de abordagem da temática. É com base em tais estudos que se apresenta um conjunto de peculiaridades e tendências atinentes às avaliações municipais.

Palavras-chave: avaliação; iniciativas municipais de avaliação; produção científica.
\end{abstract}

\section{MUNICIPAL EVALUATIONS: A BIBLIOGRAPHIC REVIEW (1990-2016)}

\section{Abstract}

This study was developed with the goal of locating and analyzing the scientific production of knowledge about municipal evaluation initiatives carried out in five specialized sources in educational evaluation, from 1990 to 2016, with a view to highlighting the main characteristic aspects of these evaluations. In the databases searched, it was identified, a total of eighteen publications were found, which contain in their titles some indicative approach to the theme. Based on such studies that the research presents a set of peculiarities and tendencies regarding the own evaluations of the municipalities.

Key-words: evaluation; municipal evaluation initiatives; scientific production.

${ }^{1}$ Universidade do Estado do Rio Grande do Norte, Brasil. E-mail: brun.silwa@gmail.com.

2 Universidade do Estado do Rio Grande do Norte, Brasil. E-mail: beatrizfernandes98@hotmail.com.

\begin{tabular}{|l|l|l|l|l|r|} 
Regae: Rev. Gest. Aval. Educ. & Santa Maria & v. 8 & n. 17 & Pub. contínua 2019 & p. 1-22
\end{tabular}




\section{Introdução}

história das avaliações externas no ensino fundamental brasileiro pode ser
narrada em três níveis: nacional, estadual e municipal, pois assim como a
União e os Estados, os municípios têm implantado suas avaliações próprias.

Foi a partir de 2005, quando o governo federal passou a avaliar a qualidade da educação com mais intensidade por meio da Prova Brasil, que o movimento de implantação de avaliações municipais se aqueceu consideravelmente. De acordo com Gatti (2009), mesmo que não tenha perdurado, uma avaliação dessa natureza foi realizada pelo município de São Paulo em 1980.

Embora não se possa afiançar de modo categórico, ao que parece, nos anos 1980 essa prática ficou restrita ao município paulistano, sendo que outras poucas municipalidades aderiram na década de 1990. Indícios desse fato foram obtidos nos estudos de Moreira (2004) e de Marques, Oliveira e Fonseca (2010) que, respectivamente, se reportam à criação dessas iniciativas pelos municípios de Três Lagoas/MS e de São Gonçalo/CE, em 1997 e em 1999. Enquanto nesses anos a existência de tais mecanismos no âmbito dos municípios era bastante rara, hoje a realidade é outra.

A esse respeito, com base nos resultados parciais da pesquisa Avaliação e gestão educacional em municípios brasileiros: mapeamento e caracterização de iniciativas em curso $^{3}$, Bauer et al. (2015) revelam que dos 4.309 municípios brasileiros participantes do referido levantamento, 1.573 já possuíam avaliação própria e 905 das redes de ensino manifestaram o propósito de implantar. Nesse sentido, a presença ou a intenção de desenvolver um sistema de avaliação próprio foram evidenciadas em mais da metade dos municípios, indicando que essas avaliações têm ganhado progressiva legitimidade na gestão municipal do ensino (Bauer et al., 2015).

Pela novidade do tema, os estudos produzidos em torno das iniciativas municipais de avaliação constituem-se preciosas fontes de informação sobre o assunto, servindo para embasar discussões e subsidiar a elaboração de variados pontos de vista. Isso posto, a presente investigação foi desenvolvida com o escopo de localizar e analisar a produção científica do conhecimento sobre o tema veiculada em cinco fontes especializadas em avaliação educacional, com a finalidade de descortinar os principais aspectos que têm caracterizado essas avaliações.

Por meio desse exercício o estudo de caráter bibliográfico proporciona maior conhecimento acerca da temática, contribuindo para o debate em torno das proposições municipais de avaliação, que nos próximos anos poderão ser cada vez mais comumente evidenciadas entre os municípios brasileiros: debate que não pode ser ignorado, já que implicações para a realidade educacional vêm a reboque da adoção desse tipo de estratégia pelas redes de ensino. Assim sendo, o estudo assume relevância pelo contributo teórico que oferece, também reconhecendo o movimento de criação de avaliações municipais como um fenômeno que instaura um novo capítulo na história das avaliações externas no Brasil, até então esboçada a partir dos sistemas nacionais e estaduais de avaliação.

3 Pesquisa conduzida pela Fundação Carlos Chagas e pelo Instituto Nacional de Estudos e Pesquisas Educacionais Anísio Teixeira, sob a coordenação dos pesquisadores Adriana Bauer e João Luiz Horta Neto. 


\section{Procedimentos metodológicos}

Para lograr o objetivo proposto, a tônica da pesquisa bibliográfica situou-se na abordagem qualitativa. Quanto à demarcação do período, de 1990 até 2016, usou-se o seguinte critério: o ano de 1990 foi definido em função de iniciar uma década marcadamente favorável ao surgimento das avaliações externas no território brasileiro. $\mathrm{O}$ levantamento estendeu-se até 2016, uma vez que a busca pelos estudos foi realizada no segundo semestre daquele ano.

Para a realização do trabalho, seguiu-se as seguintes etapas: 1 ) levantamento da produção científica relativa às iniciativas municipais de avaliação veiculada em cinco fontes especializadas em avaliação educacional, no período de 1990 a 2016; 2) leitura e fichamento das publicações com ênfase para os aspectos revelados acerca das avaliações próprias dos municípios; 3 ) análise de conteúdo do material obtido por meio dos fichamentos dos estudos.

O levantamento sistemático da produção sobre o referido tema foi realizado em cinco fontes de pesquisa: anais do Congresso Nacional de Avaliação em Educação Conave -; anais do Congresso Internacional em Avaliação Educacional - Ciae -; Revista Estudos em Avaliação Educacional; Revista Ensaio: Avaliação e Políticas Públicas em Educação; Revista Cadernos de Pesquisa. A escolha dos anais do Congresso Nacional de Avaliação em Educação e do Congresso Internacional em Avaliação Educacional foi motivada pelo fato de se constituírem como dois congressos brasileiros especializados em avaliação educacional. Por seu turno, a escolha dos periódicos teve como referência a pesquisa de Barreto e Pinto (2001), que destacou, respectivamente, a Revista Ensaio, a Revista Estudos em Avaliação Educacional e a Revista Cadernos de Pesquisa como as três maiores produtoras de trabalhos acerca da avaliação na educação básica entre os anos de 1990 a 1998.

Foram consultados: os anais do I, II, III e IV Conave, que datam respectivamente dos anos de 2010, 2012, 2014 e 2016; os anais do I, II, III, IV, V e VI Ciae, respectivamente publicados nos anos de 2004, 2005, 2006, 2008, 2010 e 2015; os números da Revista Estudos em Avaliação Educacional, do n. 1 (1990) ao n. 66 (2016); os números da Revista Ensaio: Avaliação e Políticas Públicas em Educação, do n. 1 (1993) ao n. 93 (2016); os números da Cadernos de Pesquisa, do n. 72 (1990) ao n. 162 (2016).

Nesse processo, foi identificado um total de 18 produções que contêm em seus títulos algum indicativo de abordagem da temática, especialmente uma das seguintes expressões: avaliação própria, avaliação municipal, avaliação do município, avaliação da rede municipal, avaliação do ensino fundamental. De 1990 a 2016 as primeiras investigações com foco específico em iniciativas municipais de avaliação datam do ano de 2006, como pode ser observado no quadro 1. Além dos anos de cada publicação, o quadro a seguir também apresenta a autoria dos estudos localizados nas bases de dados supracitadas e o respectivo código atribuído para cada produção. 
Quadro 1 -

Ano de publicação, autoria e código dos estudos encontrados.

\begin{tabular}{|c|c|c|}
\hline Ano & Autoria & Código \\
\hline 2006 & Costa; Oliveira & A \\
\hline 2006 & Santos; Amorim Neto & B \\
\hline 2008 & Marques; Ribeiro; Ciasca & C \\
\hline 2010 & Catalani; Gonsales & D \\
\hline 2010 & Evaristo; Santos & E \\
\hline 2010 & Lima et al. & G \\
\hline 2010 & Maroja & H \\
\hline 2010 & Marques; Barroso; Sousa & I \\
\hline 2012 & Sousa; Pimenta; Machado & J \\
\hline 2013 & Alavarse; Bravo; Machado & L \\
\hline 2013 & Gimenes et al. & N \\
\hline 2014 & Aguiar & O \\
\hline 2015 & Bauer & P \\
\hline 2015 & Bauer et al. & Q \\
\hline 2015 & Muniz; Braga; Muniz & R \\
\hline 2016 & Breves; Monteconrado; Rocha & S \\
\hline 2016 & Castro; Siqueira & Nunes; Silva \\
\hline 2016 & &
\end{tabular}

Fonte: as autoras (2018).

A partir do levantamento efetivado, procedeu-se à leitura analítica do material e ao fichamento de todos os estudos listados no quadro acima. A conclusão dessas duas etapas foi essencial para a continuidade da pesquisa, pois os fichamentos realizados serviram de subsídio para a análise das tendências atinentes às avaliações desenvolvidas na rede municipal.

\section{Avaliações municipais: principais elementos descortinados}

Por meio da leitura atenta dos objetivos dos estudos examinados, observou-se que a maioria dos trabalhos concentrou a atenção na avaliação municipal em si, preocupandose com a implementação dessas iniciativas e com as características assumidas por elas. Além dessa tendência proeminente, os estudiosos ampliaram suas análises para outras inquietações relacionadas às avaliações próprias, investigando os objetivos e justificativas que dão sustento à criação e à manutenção das avaliações municipais, as implicações dessas avaliações para o currículo escolar, os usos que têm sido feitos das informações geradas por essas avaliações, o vínculo desses mecanismos com as avaliações externas desenvolvidas em maior escala e com a melhoria da qualidade da educação.

Como veremos a partir do próximo tópico, os estudos, de um modo geral, apresentam valiosas informações acerca das experiências municipais de avaliação. Cada produção examinada, mesmo que algumas delas não tenham tido essa intenção imediata,

${ }^{4} \mathrm{O}$ trabalho, apesar de constar nos anais do evento, não estava disponível nem para download nem para leitura. 
concorre ativamente para a construção do conhecimento relativo a esse tema. Se cada trabalho já é de grande relevância, a reunião, sistematização e análise dos elementos apontados no âmbito de cada estudo contribui ainda mais para o alcance de níveis elevados de compreensão em torno das avaliações municipais.

\section{Quanto à denominação}

Os estudos consultados, sobretudo os que analisam somente um caso, apresentam o nome das iniciativas municipais de avaliação. Tal informação foi parcialmente obtida nos trabalhos de Alavarse, Bravo e Machado (2013); Gimenes et al. (2013); e Sousa, Pimenta e Machado (2012), que não citaram o nome de todas as avaliações estudadas. Também convém destacar que evidências a esse respeito só não foram extraídas dos estudos de Bauer et al. (2015) e de Marques, Ribeiro e Ciasca (2008).

Isso posto, foram identificadas as seguintes denominações: Avaliação da Aprendizagem Escolar da Rede Pública Municipal de Ensino de Serrinha/BA - Avapre; Avaliação de Desempenho do Estudante - ADE; Avaliação do Desempenho Escolar de Alunos da Rede Municipal de Angra dos Reis, Avaliação Municipal da Educação de Tauá Amet; Política de Avaliação da Rede Municipal de Ensino de Petrolina - Parmep; Programa de Verificação do Rendimento Escolar da Rede Municipal de Ensino - Prove; Prova da Cidade, Prova São Paulo, Sistema de Avaliação da Educação Municipal de Juazeiro - Saej; Sistema de Avaliação da Educação Municipal em Jijoca de Jericoacoara Saemjj; Sistema de Avaliação da Secretaria de Educação de Maracanaú, Sistema de Avaliação Municipal da Educação de Barrinha - Sameb; Sistema de Avaliação de Aproveitamento Escolar dos alunos da Rede Municipal de Ensino de São Paulo, Sistema de Avaliação de Desempenho Educacional de Manaus - Sadem; Sistema de Avaliação do Rendimento Escolar do Município de Catanduva - Saremca.

As iniciativas cujos nomes foram mencionados, possuem designativos bem definidos, sendo que a maioria incorpora o nome do município. Isso, em nossa ótica, além de ser um indício de que tais avaliações são de significativa relevância para a gestão educacional dos municípios, ao ponto de levar o nome do município, sinalizam que a criação das mesmas foi objeto de idealização e planejamento por parte das secretarias municipais de educação.

\section{Quanto ao ano de criação}

Os estudos também apresentaram informações acerca dos anos de criação das avaliações municipais investigadas. Alguns dos estudos inventariados investigaram mais de uma avaliação, logo, ressaltaram mais de um ano de implementação. Sousa, Pimenta e Machado (2012), por exemplo, relatam que as avaliações próprias estudadas foram implementadas no ano de 2005, salientando, porém, que a avaliação do município de Marília/SP já havia sido criada em 2004.

Dentre os estudos analisados apenas um não destacou o ano de implementação da avaliação municipal analisada, sendo o estudo de Lima et al. (2010). Todos os demais enfatizaram o ano de implantação da avaliação própria, compreendido em um dos períodos a seguir. 
Tabela 1 -

Ano de implementação das avaliações.

\begin{tabular}{c|c|c}
\hline Período & Estudo & Total \\
\hline Anterior a 2000 & O & 1 \\
\hline 2000 a 2004 & A, B, I, O & 4 \\
\hline 2005 a 2009 & D, E, C, G, H, I, J, L, O, P, R & 11 \\
\hline 2010 a 2014 & M, O, Q, S & 4 \\
\hline
\end{tabular}

Fonte: as autoras (2018).

Como pode-se notar, um dos estudos analisados localizou a existência dessas avaliações antes mesmo dos anos 2000. Nesse período, conforme Bauer et al. (2015), foram criadas um total de 34 avaliações pelos municípios. Apesar de não revelar com precisão o ano, nem o nome dos municípios, Bauer et al. (2015) destacam que a primeira avaliação dessa natureza foi criada no limiar da década de 1980, reforçando, desse modo, o relato tecido por Gatti (2009) concernente à avaliação municipal da cidade de São Paulo. Bauer et al. (2015) destacaram ainda que a implantação dessas iniciativas se tornou mais frequente a partir de 2005. Se no ano de 2004 foram criadas cerca de 103 avaliações municipais, o intervalo de 2005 a 2014 foi palco para o surgimento de outras 1.280 avaliações. Tal tendência, a propósito, foi posta em relevo em 11 dos estudos, incluindo o de Bauer et al. (2015), que analisaram iniciativas próprias de avaliação criadas entre os anos de 2005 a 2009. Há muito sentido, portanto, quando Bauer, Horta Neto e Sousa (2016) afirmam que

é principalmente a partir de 2005 que a criação de avaliações próprias pelos municípios se intensifica. Parece haver correspondência entre a sua crescente presença e as iniciativas do Ministério da Educação, sejam aquelas relativas à avaliação em larga escala, seja a criação de índice para se aquilatar o desenvolvimento da qualidade educacional (Ideb), que pode ter influenciado o crescente movimento de apropriação dos resultados das provas por gestores municipais. (p. 129)

Ao que parece, o surgimento das avaliações municipais está bastante relacionado com o Sistema Nacional de Avaliação da Educação Básica - SAEB, cuja influência sobre as redes de ensino passa a ser exercida com maior força a partir desse período, em decorrência da criação da Prova Brasil em 2005 e do Índice de Desenvolvimento da Educação Básica - Ideb em 2007. Isso posto, é bem provável que as avaliações municipais implantadas a partir de então, tenham sido concebidas como uma estratégia para potencializar o aprendizado dos alunos em Língua Portuguesa e em Matemática, com vistas a obter melhores resultados na Prova Brasil e, assim, atender as expectativas impostas pelo Ideb. 


\section{Quanto à referência seguida para a elaboração da avaliação municipal}

Um aspecto mencionado em sete dos estudos analisados e que também merece destaque é a referência seguida para elaboração do modelo de avaliação municipal. Com base nas produções visitadas, as iniciativas municipais de avaliação se espelham nas avaliações externas e em larga escala já existentes, principalmente naquelas desenvolvidas pela União, como é possível analisar na tabela abaixo:

Tabela 2 -

Referência seguida para a elaboração da avaliação municipal.

\begin{tabular}{c|c|c}
\hline Referência & Estudos & Total \\
\hline SAEB/Prova Brasil & L, H, P, S & 4 \\
\hline Provinha Brasil & D, E & 2 \\
\hline SPAECE & $\mathrm{H}, \mathrm{P}, \mathrm{H}$ & 3 \\
\hline Prova do PAIC & $\mathrm{H}$ & 1 \\
\hline Não especifica & $\mathrm{I}$ & 1 \\
\hline Não menciona & $\mathrm{A}, \mathrm{B}, \mathrm{C}, \mathrm{F}, \mathrm{G}, \mathrm{J}, \mathrm{M}, \mathrm{O}, \mathrm{Q}, \mathrm{R}$ & 10 \\
\hline
\end{tabular}

Fonte: as autoras (2018).

No estudo de Sousa, Pimenta e Machado (2012), mesmo que não haja uma especificação da avaliação tomada como referência, as autoras são enfáticas em afirmar que os sistemas nacionais e estaduais têm sido utilizados como parâmetro para a proposição das avaliações municipais. Esse, claramente, também foi o caso das avaliações dos municípios cearenses estudadas por Muniz, Braga e Muniz (2015) e por Marques, Barroso e Sousa (2010), que além da Prova Brasil também tomaram o sistema de avaliação do estado do Ceará, Spaece, como arquétipo. Essa também foi uma das conclusões obtidas por Ovando (2011), após analisar as experiências avaliativas desenvolvidas por quatro municípios sul-mato-grossenses: "a avaliação municipal tem se dado de forma reprodutora, seguindo os moldes das avaliações nacionais, restrita nos aspectos metodológicos" (p. 97).

Apesar de boa parte dos trabalhos não terem apontado essa tendência, há grandes chances de que tal alinhamento seja feito com bastante frequência pelas secretarias municipais de educação. As razões para tanto podem ser de variadas ordens, mas pode ser indício de que as avaliações municipais têm sido engendradas como um subterfúgio para a preparação dos estudantes para as avaliações externas à rede de ensino, tomadas como referência. Também é possível que as secretarias usem os modelos avaliativos em vigor como ponto de partida, fazendo um estudo de suas limitações e potencialidades e, sobretudo, buscando que suas avaliações superem as restrições comuns a esses sistemas de maior escala e atendam, de fato, às necessidades dos gestores, coordenadores, professores e alunos.

Ainda em relação a esse assunto convém fazer um destaque para o que se constatou no estudo de Nunes e Silva (2016) que, na condição de integrantes da secretaria municipal de educação investigada, informam que havia nos propositores da avaliação o desejo de criar uma avaliação com suas peculiaridades próprias, sem intenção de imitar os sistemas avaliativos em vigor mas, todavia, a Prova Brasil foi 
tomada como "referência para construção dos instrumentos e seleção das habilidades a serem avaliadas" (Nunes; Silva, 2016, p. 2). Nesses termos, o desiderato de desenhar uma avaliação autêntica foi abandonado ou, na melhor das hipóteses, parcialmente atendido.

\section{Quanto à formalização legal}

Em alguns dos estudos, pode-se localizar informações acerca do reconhecimento legal da avaliação própria do município. Evidências disso foram obtidas nos trabalhos de Aguiar (2014), Alavarse, Bravo e Machado (2013); Breves, Monteconrado e Rocha (2016); Gimenes et al. (2013); Marques, Barroso e Sousa (2010); e Nunes e Silva (2016), que fazem menção a determinados dispositivos - lei, decreto ou resolução - de cunho municipal, regendo as iniciativas próprias de avaliação de Juazeiro/BA, Catanduva/SP, Manaus/AM, São Paulo/SP, Maracanaú/CE e Serrinha/BA, respectivamente.

Além desses estudos, apontamentos a esse respeito também foram efetivados por Bauer et al. (2015). De acordo com tais estudiosos das 1.573 avaliações municipais estudadas apenas $16 \%$ possuem regulamentação. Tais achados, na perspectiva dos referidos pesquisadores, permitem "afirmar que a realização de avaliações próprias antecede à sua formalização legal” (Bauer et al., 2015, p. 341). Nesse sentido, os primeiros anos de realização da avaliação municipal atuam como um balão de ensaio para se decidir em relação ao reconhecimento legal da avaliação pelo sistema de ensino. Isso, porém, não deve ser tomado como um denominador comum a todas as avaliações municipais, embora seja um percurso bastante trilhado. É provável que algumas municipalidades tenham dado esse passo concomitantemente ao ano de criação da avaliação. Ademais, também é possível que certas iniciativas tenham suas histórias iniciadas e concluídas sem nunca terem passado por esse processo.

\section{Quanto aos objetivos almejados}

Os trabalhos consultados, cada um a seu modo, expõem os objetivos que as secretarias municipais têm buscado alcançar por meio e a partir da avaliação municipal. Os objetivos identificados, além de numerosos, são bastante diversificados, revelando muito acerca do sentido em que são empregadas as avaliações próprias. As informações referentes a esse aspecto foram facilmente identificadas na maioria dos estudos. Alguns poucos trabalhos, porém, apresentam esse dado de forma sutil, sem muito destaque. Com base nesse material, chegou-se a um conjunto de objetivos, aqui apresentado em quatro blocos.

Em primeiro lugar discerniu-se que alguns dos objetivos das secretarias relacionavam-se com a ideia de conhecer, diagnosticar e obter dados da realidade educacional. Para perceber tal semelhança de modo mais inteligível, esse subconjunto de objetivos foi, intencionalmente, reunido em um único bloco.

Tabela 3 -

Objetivos atinentes à obtenção de um diagnóstico.

\begin{tabular}{l|l|c}
\hline \multicolumn{1}{c|}{ Objetivo } & \multicolumn{1}{c|}{ Estudos } & Total \\
\hline $\begin{array}{l}\text { Avaliar o desempenho acadêmico dos } \\
\text { alunos }\end{array}$ & $\begin{array}{l}\text { A, B, C, D, E, F, G, H, I, } \\
\text { J, M, O, Q, S }\end{array}$ & 14 \\
\hline
\end{tabular}




\begin{tabular}{l|l|c}
\hline $\begin{array}{l}\text { Acompanhar/monitorar o processo de } \\
\text { ensino-aprendizagem }\end{array}$ & F, I, P, Q, S & 5 \\
\hline Diagnosticar as dificuldades dos alunos & C, D, E, H, R & 5 \\
\hline $\begin{array}{l}\text { Gerar um diagnóstico da educação } \\
\text { municipal }\end{array}$ & $\mathrm{H}, \mathrm{J}, \mathrm{Q}$ & 3 \\
\hline $\begin{array}{l}\text { Discriminar os resultados da avaliação por } \\
\text { escola, por turma e por alunos }\end{array}$ & $\mathrm{L}$ & 1 \\
\hline $\begin{array}{l}\text { Produzir informações atinentes aos } \\
\text { alunos, professores, coordenadores, } \\
\text { diretores e escolas }\end{array}$ & $\mathrm{F}$ & 1 \\
\hline
\end{tabular}

Fonte: as autoras (2018).

Nesse bloco, sobressai o anseio de tomar conhecimento da situação de aprendizagem dos estudantes tendo como referência o desempenho dos mesmos no teste ou no instrumento avaliativo utilizado. Algumas municipalidades, convém destacar, têm sido bem específicas no tocante a essa intenção, buscando conhecer não o que os alunos aprenderam, mas as lacunas no aprendizado. A secretaria municipal de São Paulo/SP, por exemplo, visa, por meio da avaliação própria, a "descrever e analisar as dificuldades dos alunos em relação a determinadas habilidades, nos seus diferentes níveis de complexidade" (Castro; Siqueira, 2016, p. 2).

Ainda com base na performance dos alunos, determinadas secretarias também têm buscado obter indicativos do ensino ofertado. Neste sentido, num dos trabalhos destacase que, a avaliação municipal associa-se à necessidade de "conhecer, efetivamente, como a escola vem desempenhando o seu papel, como os alunos estão aprendendo" (Nunes; Silva, 2016, p. 3). Similarmente, no município de Tauá/CE, a avaliação criada para proporcionar tanto o diagnóstico quanto o "acompanhamento da aprendizagem dos alunos e das práticas educativas dos sujeitos" (Lima et al., 2010, p. 2444). Diante de tal finalidade, vale acrescentar que a aprendizagem avaliada guarda estreita relação com as oportunidades educacionais oferecidas.

O segundo agrupamento, por sua vez, coaduna objetivos diferenciados, mas que, apesar disso, possuem um ponto em comum. Como pode-se visualizar na tabela 4 todos os objetivos vão além do ato de diagnosticar, referindo-se ao objetivo intencionado com base no diagnóstico proporcionado pela avaliação municipal.

Tabela 4 -

Objetivos atinentes ao desenvolvimento de ações, estratégias e medidas.

\begin{tabular}{l|l|c}
\hline \multicolumn{1}{c|}{ Objetivo } & \multicolumn{1}{c|}{ Estudos } & Total \\
\hline $\begin{array}{l}\text { Subsidiar o desenvolvimento da } \\
\text { intervenção pedagógica sobre a situação } \\
\text { de aprendizagem diagnosticada }\end{array}$ & B, C, E, F, G, L, R, S & 8 \\
\hline Repensar a prática pedagógica & $\mathrm{B}, \mathrm{G}, \mathrm{H}, \mathrm{I}, \mathrm{J}, \mathrm{O}, \mathrm{Q}, \mathrm{S}$ & 8 \\
\hline $\begin{array}{l}\text { Formular, reformular e/ou acompanhar } \\
\text { políticas e programas educacionais }\end{array}$ & $\mathrm{B}, \mathrm{G}, \mathrm{H}, \mathrm{J}, \mathrm{Q}, \mathrm{S}$ & 6 \\
\hline
\end{tabular}




\begin{tabular}{l|l|c}
\hline $\begin{array}{l}\text { Viabilizar o acesso às questões e às } \\
\text { respostas dos alunos }\end{array}$ & D, E, I, L, R & 5 \\
\hline Nortear a formação continuada & G, L, O, S & 4 \\
\hline $\begin{array}{l}\text { Preparar os alunos para as avaliações de } \\
\text { maior escala }\end{array}$ & D, E, O & 3 \\
\hline $\begin{array}{l}\text { Subsidiar o planejamento dos professores } \\
\text { e gestores escolares }\end{array}$ & E, H, L & 3 \\
\hline Estabelecer e/ou acompanhar metas & J, L & 2 \\
\hline $\begin{array}{l}\text { Implantar estratégias de controle, } \\
\text { prestação de contas e responsabilização }\end{array}$ & J, O & 2 \\
\hline $\begin{array}{l}\text { Incitar maior envolvimento dos atores } \\
\text { escolares com os resultados da avaliação }\end{array}$ & I, S & 2 \\
\hline Repensar as orientações curriculares & G, S & 2 \\
\hline Apresentar informações à população & G & 1 \\
\hline $\begin{array}{l}\text { Possibilitar que as escolas se enxerguem } \\
\text { nos resultados da avaliação }\end{array}$ & F & 1 \\
\hline $\begin{array}{l}\text { Reconhecer e valorizar o esforço de } \\
\text { alunos, profissionais da educação e } \\
\text { escolas }\end{array}$ & F & 1 \\
\hline
\end{tabular}

Fonte: as autoras (2018).

Os objetivos constantes nesse bloco indicam para que o diagnóstico foi feito, apontando para o desenvolvimento de ações baseadas nos resultados da avaliação por parte da secretaria de educação e das escolas. No plano da secretaria, destaca-se o aproveitamento do diagnóstico para propor ou avaliar políticas e programas educacionais, também sendo retomado na formação continuada dos profissionais da educação da rede. Além disso, a avaliação também tem sido tomada como um instrumento de grande valia para induzir processos de prestação de contas e responsabilização dos atores escolares pelos resultados obtidos. No plano das escolas, aliás, tenciona-se que os agentes escolares se apropriem desses resultados para que possam intervir sobre os mesmos, o que também não deixa de ser uma forma de preparar os alunos para as avaliações externas, pretensão manifestada por algumas secretarias.

O terceiro agrupamento congrega objetivos referentes a determinadas configurações que as secretarias municipais de educação projetam para suas avaliações.

Tabela 5 -

Objetivos atinentes às características da avaliação municipal.

\begin{tabular}{l|l|c}
\hline \multicolumn{1}{c|}{ Objetivo } & \multicolumn{1}{|c|}{ Estudos } & Total \\
\hline $\begin{array}{l}\text { Respeitar as particularidades da realidade } \\
\text { educacional local }\end{array}$ & A, M, Q & 3 \\
\hline $\begin{array}{l}\text { Proporcionar a participação dos } \\
\text { professores na elaboração dos itens da } \\
\text { avaliação própria }\end{array}$ & D, E & 2 \\
\hline
\end{tabular}




\begin{tabular}{l|l|c}
\hline $\begin{array}{l}\text { Proporcionar a participação da } \\
\text { comunidade escolar nas etapas da } \\
\text { avaliação }\end{array}$ & R, S & 2 \\
\hline $\begin{array}{l}\text { Proporcionar o acesso aos critérios de } \\
\text { avaliação dos itens }\end{array}$ & E, R & 2 \\
\hline $\begin{array}{l}\text { Dialogar com os documentos curriculares } \\
\text { da SME }\end{array}$ & E, R & 2 \\
\hline Obter um diagnóstico censitário e/ou anual & C, L & 2 \\
\hline Ampliar o número de anos avaliados & L & 1 \\
\hline $\begin{array}{l}\text { Realçar a dimensão pedagógica da } \\
\text { avaliação }\end{array}$ & S & 1 \\
\hline
\end{tabular}

Fonte: as autoras (2018).

Como pode-se observar, as secretarias conferem às avaliações municipais características que não são localizadas dentre os sistemas de avaliação externos ao município. Nesse sentido, foi intenção da secretaria de Angra dos Reis/RJ, por exemplo, promover uma avaliação baseada "na realidade socioeconômica da região, respeitando as especificidades e as peculiaridades locais" (Costa; Oliveira, 2006, p. 150). Cumpre-se salientar que esse desejo também foi identificado em mais duas secretarias, a de Juazeiro/BA e a de Manaus/AM. No município de Serrinha/BA, de modo particular, os idealizadores da iniciativa buscaram resgatar a função pedagógica da avaliação. Segundo Nunes e Silva (2016) "buscou-se fomentar na rede, através do Sistema Avapre, a reconstrução do conceito de avaliação em uma dimensão pedagógica e reflexiva (ação, reflexão, ação refletiva)" (p. 3).

Além disso, em alguns casos, aspira-se que a avaliação seja mais próxima dos profissionais da educação, permitindo a atuação dos mesmos na proposição dos itens que irão compor o instrumento avaliativo, assim como nos diferentes estágios da avaliação. Também aparece como intenção entre as secretarias que as avaliações proporcionem diagnósticos com periodicidade menor do que a adotada pelos demais sistemas de avaliação e com maior abrangência de anos avaliados.

Os objetivos apresentados até este ponto, além da relação que estabelecem em si, têm sido traçados com vistas a alcançar finalidades educacionais mais amplas, ou seja, o que, de fato, se espera conseguir. Por esse motivo, optou-se por apresentar tais objetivos em um bloco específico.

Tabela 6 -

Objetivos atinentes a finalidades educacionais mais amplas.

\begin{tabular}{l|c|c}
\hline \multicolumn{1}{c|}{ Objetivo } & Estudos & Total \\
\hline $\begin{array}{l}\text { Melhorar a qualidade da aprendizagem, } \\
\text { do ensino ou da educação }\end{array}$ & A, B, C, G, H, J, O, P, Q, S & 10 \\
\hline Melhorar índices educacionais & B, O, S & 3 \\
\hline Melhorar a gestão das escolas & G, S & 2 \\
\hline Combater o analfabetismo & $\mathrm{C}$ & 1 \\
\hline
\end{tabular}

Fonte: as autoras (2018). 
É de destaque a expectativa de que se lograr uma melhoria da qualidade, sendo que alguns municípios fazem menção à melhoria da qualidade da educação e outros à melhoria da qualidade da aprendizagem ou do ensino. Imersas nessa mesma lógica, determinadas secretarias buscam, com os esforços e investimentos empreendidos, melhorar - elevar ou reduzir - índices educacionais, especialmente o ldeb.

Tendo em vista tais objetivos, não é demais dizer que as municipalidades têm depositado grandes expectativas nessas iniciativas. Em virtude disso é provável que alguns deles circunscrevam-se apenas ao plano das intenções, ficando longe de se efetivarem na prática.

\section{Quanto ao foco da avaliação}

Como se sabe, em educação, a instituição de qualquer avaliação passa pela escolha de quem será o público avaliado. Assim como os sistemas de avaliação realizados em maior escala, as avaliações promovidas pelos municípios podem direcionar seu interesse para o aluno, o professor, o diretor ou, até mesmo, para a escola de um modo geral.

De acordo com as publicações consultadas, as iniciativas municipais de avaliação estão proeminentemente preocupadas com o aluno. Para ser mais preciso, a maioria delas avalia o aluno de modo específico: fugiram dessa regra apenas as avaliações estudadas por Alavarse, Bravo e Machado (2013) e por Sousa, Pimenta e Machado (2012), que além do aluno também realizam avaliação do professor. Tal constatação serve como um indicativo de que o principal ou único fator considerado pelas municipalidades é o desempenho dos estudantes, no caso, o desempenho em Língua Portuguesa e em Matemática, como veremos mais adiante.

Ao assumir tal postura, os municípios, por meio de suas avaliações próprias, colaboram para o reordenamento do currículo escolar em função das avaliações externas. Como explica Coutinho (2012), "o currículo que é posto em prática por professores é aquele que será objeto de avaliação, acarretando a seleção de determinados tipos de conteúdos em detrimento da exclusão de outros" (p. 23). No plano dos municípios, a opção por avaliar apenas Português e Matemática soa como um comando, direto ou indireto, da própria secretaria municipal de educação para que haja uma priorização de ambas as áreas do saber. Com isso, até mesmo por conta da maior proximidade com a rede de ensino, as consequências sobre o currículo são quase que inevitáveis.

\section{Quanto aos anos do ensino fundamental avaliados}

Outro ponto bastante destacado na maioria dos trabalhos trata dos anos do ensino fundamental abrangidos pela avaliação municipal. Com base nas informações presentes nas publicações examinadas, pode-se discriminar duas tendências. Em primeiro lugar evidenciou-se a tendência de avaliar os mesmos anos avaliados pelo Saeb e pela maioria dos sistemas estaduais de avaliação. A preocupação específica com tais anos - $2^{\circ}, 5^{\circ}$ ou 9o - foi mencionada por Marques, Ribeiro e Ciasca (2008); Costa e Oliveira (2006); e Sousa, Pimenta e Machado (2012). O foco nesses anos coaduna com a ideia de que as avaliações municipais são criadas com o objetivo de melhorar os índices das avaliações estaduais e nacionais, justificando assim a opção por esses anos. 
A segunda tendência entre os municípios consiste em monitorar todos, ou praticamente todos, os anos do ensino fundamental por meio da avaliação própria. Tal disposição foi observada a partir dos apontamentos efetivados por Aguiar (2014); Breves, Monteconrado e Rocha (2016); Gimenes et al. (2013); Lima et al. (2010); Sousa, Pimenta e Machado (2012). Vale destacar, oportunamente, que a intenção de estender a avaliação para os demais anos do ensino fundamental foi destacada na municipalidade analisada por Marques, Barroso e Sousa (2010).

No entanto, de modo geral, percebe-se que a escolha das séries para realização dos testes não segue um padrão. Dito de outro modo, os anos do ensino fundamental submetidos à avaliação própria variam muito de município para município. Os estudos revelam irregularidades quanto aos anos selecionados para avaliação. Uma das avaliações próprias estudadas por Gimenes et al. (2013), por exemplo, focou no $3^{\circ}$, $4^{\circ} \mathrm{e}$ 50 ano, já a avaliação investigada por Catalani e Gonsales (2010); Evaristo e Santos (2010); e Maroja (2010) optou por avaliar a $2^{a}$, $4^{a}, 6^{a}$ e $8^{a}$ série, $3^{o}, 5^{o}, 7^{o}$ e $9^{o}$ ano, respectivamente.

\section{Quanto às áreas do conhecimento avaliadas}

A maioria dos estudos também se preocupou em mencionar as áreas de conhecimentos avaliadas pelos municípios. Abriu-se espaço para tanto nos seguintes estudos: Aguiar (2014); Breves, Monteconrado e Rocha (2016); Castro e Siqueira (2016); Catalani e Gonsales (2010); Costa e Oliveira (2006); Evaristo e Santos (2010); Lima et al. (2010); Maroja (2010); Marques, Barroso e Sousa (2010); Marques, Ribeiro e Ciasca (2008); Santos e Amorim Neto (2006); Sousa, Pimenta e Machado (2012).

De acordo com tais investigações, as iniciativas municipais avaliam duas áreas do saber, Língua Portuguesa e Matemática, com exceção apenas das avaliações estudadas por Breves, Monteconrado e Rocha (2016) e por Marques, Ribeiro e Ciasca (2008), que não se prendem a essas duas disciplinas especificamente. $O$ modelo de avaliação apresentado por Marques, Ribeiro e Ciasca (2008), à época da realização do estudo, avaliava a aquisição e apropriação da leitura e escrita (alfabetização), ou seja, apenas a área de Língua Portuguesa. Já a avaliação municipal estudada por Breves, Monteconrado e Rocha (2016), além de Português e Matemática, avaliava os conhecimentos dos estudantes em Ciências da Natureza e Humanidades.

A opção dos municípios por voltar suas avaliações para o desempenho dos estudantes em Língua Portuguesa e Matemática é interpretada como um indício do enlace das proposições de avaliação municipal com as demais avaliações externas. As proficiências dos alunos em ambas as áreas é um dos elementos determinantes do Ideb, usado como sinônimo da qualidade da educação ofertada no país.

\section{Quanto ao referencial curricular}

Em boa parte das investigações os pesquisadores apresentam suas descobertas acerca do referencial curricular adotado pelas avaliações municipais. 
Tabela 7 -

Referencial curricular utilizado nas avaliações municipais.

\begin{tabular}{l|c|c}
\hline \multicolumn{1}{c|}{ Objetivo } & Estudos & Total \\
\hline Referencial curricular & Estudos & Total \\
\hline Documento de cunho municipal & D, E, F, G, P, S & 6 \\
\hline Documento de cunho nacional & $\mathrm{A}$ & 1 \\
\hline Documento de cunho estadual & $\mathrm{H}$ & 1 \\
\hline Documento de centro especializado & $\mathrm{C}$ & 1 \\
\hline $\begin{array}{l}\text { Documento municipal e documento de } \\
\text { cunho nacional }\end{array}$ & $\mathrm{M}, \mathrm{Q}$ & 2 \\
\hline Não menciona & $\mathrm{B}, \mathrm{I}, \mathrm{J}, \mathrm{L}, \mathrm{O}, \mathrm{R}$ & 6 \\
\hline Total & & 17 \\
\hline
\end{tabular}

Fonte: as autoras (2018).

Como pode-se notar, documentos de cunho municipal, como é o caso das propostas curriculares e das matrizes de referência definidas pelo próprio município, foram os referenciais curriculares mais frequentemente citados entre os estudos. Vale destacar que duas das investigações (Muniz; Braga; Muniz, 2015; Lima et al., 2010) revelaram que tais referências municipais foram elaboradas com base nos Parâmetros Curriculares Nacionais e nas matrizes do Sistema Permanente de Avaliação da Educação Básica do Ceará - Spaece. Além das matrizes do Spaece, estudada por Muniz; Braga; Muniz (2015) as matrizes de referência da Prova Brasil também foram úteis para essa elaboração.

Em outras realidades o uso específico de referenciais municipais também pode estar associado à utilização de outros documentos de abrangência nacional: diretrizes curriculares nacionais e matrizes de referência da Avaliação Nacional de Alfabetização e da Prova Brasil, como foi destacado por Aguiar (2014); Breves, Monteconrado e Rocha (2016).

Três dos estudos também mencionaram o uso específico de referências externas ao município, como as matrizes do Sistema de Avaliação da Educação Básica (Costa; Oliveira, 2006), a matriz de referência em alfabetização do estado do Ceará (Marques; Barroso; Sousa, 2010) e a matriz de alfabetização do Centro de Alfabetização, Leitura e Escrita da Universidade Federal de Minas Gerais (Marques; Ribeiro; Ciasca, 2008).

\section{Quanto à periodicidade}

A periodicidade das iniciativas municipais de avaliação foi revelada em seis dos 17 estudos inventariados. Mais precisamente foram os estudos de Aguiar (2014), Alavarse, Bravo e Machado (2013); Gimenes et al. (2013); Marques, Ribeiro e Ciasca (2008); Muniz, Braga e Muniz (2015); e Sousa, Pimenta e Machado (2012) que contribuíram para a produção do conhecimento acerca desse importante aspecto. Tais estudos registram que as avaliações municipais têm frequência anual, sendo realizadas uma, duas ou até três vezes ao ano. Dentre essa frequência, a aplicação da avaliação duas vezes durante $o$ ano foi a que mais se sobressaiu dentre as secretarias estudadas por tais pesquisadores. 
Além disso, convém destacar que quando realizada apenas uma vez os gestores priorizam sua realização no final do ano letivo. Se realizada duas vezes é possível que seja aplicada no início e no final do ano ou no fim de cada semestre letivo. Já quando se realiza três vezes, tende a ocorrer no início, no meio e no final do ano.

Com isso, não é demais dizer que, por meio de suas avaliações próprias, as secretarias estão, periodicamente, à par da situação de aprendizagem dos alunos. Resta saber se é feito o devido uso das informações obtidas com vistas a melhoria do aprendizado diagnosticado, evitando, assim, que a avaliação municipal resulte, apenas, em mais uma constatação.

\section{Quanto ao instrumental utilizado}

Outro aspecto bastante mencionado pelos estudos trata do instrumental utilizado nas avaliações próprias. Todas as iniciativas municipais de avaliação abordadas nas investigações utilizam-se do teste, ou prova, como é popularmente conhecido.

Além do teste algumas das avaliações municipais também fazem uso do questionário. Na avaliação de Barrinha/SP, estudado por Sousa, Pimenta e Machado (2012), o questionário é utilizado para a avaliação do professor. Já na avaliação analisada por Costa e Oliveira (2006), esse recurso é usado para obter informações referentes ao aluno e à escola, ademais, a rede também realiza entrevista com os diretores e professores de Língua Portuguesa e Matemática, lotados no $5^{\circ}$ e do $9^{\circ}$ ano do ensino fundamental. Aguiar (2014), por sua vez, não especifica para quem o instrumento é destinado.

\section{Quanto ao pessoal envolvido na realização da avaliação}

A partir da leitura acurada das produções também se extraiu valiosas informações relativas ao pessoal envolvido na realização da avaliação municipal. Como pode-se observar na tabela 8 as investigações apontaram com maior frequência a responsabilidade dos técnicos da Secretaria Municipal de Educação pela condução desse processo. Boa parte desses estudos (Breves, Monteconrado e Rocha, 2016; Catalani e Gonsales, 2010; Maroja, 2010; Muniz; Braga; Muniz, 2015; Nunes; Silva, 2016) menciona, inclusive, que nas municipalidades investigadas, tais técnicos se organizam em um setor criado especificamente para dar conta dessa tarefa.

Tabela 8 -

Pessoal envolvido na realização da avaliação.

\begin{tabular}{c|c|c}
\hline $\begin{array}{c}\text { Pessoal envolvido na realização da } \\
\text { avaliação }\end{array}$ & Estudos & Total \\
\hline Técnicos da Secretaria de Educação & C, D, G, I, J, O, P, Q, S & 9 \\
\hline Professores & D, E, I, J, L, O, R & 7 \\
\hline Assessoria externa & $\mathrm{A}, \mathrm{C}, \mathrm{I}, \mathrm{J}, \mathrm{L}, \mathrm{O}$ & 6 \\
\hline Gestores escolares & $\mathrm{I}, \mathrm{J}, \mathrm{L}, \mathrm{O}$ & 4 \\
\hline Pais e alunos & $\mathrm{J}$ & 1 \\
\hline Não menciona & $\mathrm{B}, \mathrm{F}, \mathrm{H}, \mathrm{M}$ & 4 \\
\hline
\end{tabular}

Fonte: as autoras (2018). 
A participação de professores também foi bastante expressiva, sendo citada em sete dos estudos. Os professores atuam nos processos de elaboração, aplicação e correção dos instrumentos avaliativos. Essas duas últimas etapas, aplicação e correção, também contam com a colaboração de gestores escolares. Além desses atores o estudo de Alavarse, Bravo e Machado (2013) registra o envolvimento de pais e alunos na avaliação. Nessa perspectiva, com a incorporação de diferentes atores educacionais nas etapas da avaliação, as secretarias de educação pautam suas propostas de avaliação em bases

mais participativas. Tal envolvimento também se torna conveniente às secretarias por contribuir na redução de gastos para a realização da avaliação. Por meio dessa abertura, os municípios podem, inclusive, promover a avaliação autonomamente, sem precisar de contratação de pessoal ou de serviço especializado.

Alguns das investigações sinalizaram que as secretarias estudadas têm contado com o apoio de assessoria externa para que a avaliação própria seja realizada. De acordo com os estudos consultados, tal assessoria foi prestada por empresas, institutos e universidades, como é o caso do Instituto Ayrton Senna, do Sistema COC de Ensino, do Centro de Políticas Públicas e Avaliação da Educação da Universidade Federal de Juiz de Fora e do Núcleo de Gestão e Avaliação da Universidade do Estado do Rio de Janeiro. Quanto a isso os estudos mencionaram a intervenção da assessoria externa em diferentes estágios da avaliação, especificamente na implantação do sistema de avaliação, na elaboração e aplicação dos testes, na análise dos dados ou, até mesmo, na realização da avaliação em sua totalidade.

A atuação de agentes externos na promoção da avaliação municipal é, inclusive, algo que merece ser investigado e discutido em maior profundidade, analisando, por exemplo, se são as secretarias que buscam esse suporte externo ou se são esses agentes que oferecem seus serviços às secretarias, tanto para as que já possuem, quanto para as que ainda não detêm esse tipo de iniciativa, seduzindo-as a criarem suas avaliações próprias.

\section{Quanto ao método e tempo destinado à análise de dados}

Alguns dos estudos também informam acerca do método utilizado pelas secretarias municipais de educação para analisar as respostas fornecidas pelos alunos aos itens do teste. Como consta da tabela a seguir, a Teoria Clássica dos Testes - TCT - e a Teoria de Resposta ao Item - TRI, mais a TCT do que a TRI, foram as duas estratégias identificadas.

Tabela 9 -

Método de análise das respostas dos alunos aos itens fechados.

\begin{tabular}{c|c|c}
\hline Método & Estudo & Total \\
\hline Teoria Clássica dos Testes (TCT) & C, D, E, G & 4 \\
\hline Teoria de Resposta ao Item (TRI) & A, G & 2 \\
\hline Não menciona & $\begin{array}{c}\text { B, F, H, I, J, L, M, O, P, Q, } \\
\text { R, S }\end{array}$ & 12 \\
\hline
\end{tabular}

Fonte: as autoras (2018). 
Inegavelmente, tais teorias são as estratégias tradicionalmente empregadas nesse tipo de análise. Dentre os sistemas avaliativos de maior escala, entretanto, a metodologia predominante é a TRI. Ao que tudo indica determinadas avaliações municipais também têm feito esse uso. Todavia, nos poucos estudos que fornecem dados relativos a esse assunto, a TCT foi o caminho mais frequentemente recorrido. Embora não seja possível afiançar, há possibilidade de que esse quadro seja uma amostra bastante representativa das avaliações municipais como um todo e que haja entre essas iniciativas uma maior adesão à TCT, em grande parte porque esse método é mais simples, sem demandar muita expertise nem para utilizá-lo nem para compreender os resultados proporcionados.

Quanto ao tempo necessário para a realização da análise dos dados foram observados esclarecimentos a esse respeito apenas nos trabalhos Aguiar (2014) e de Sousa, Pimenta e Machado (2012). De acordo com Aguiar (2014), a avaliação própria estudada tende a ser aplicada "em novembro de cada ano e os boletins entregues ao final de dezembro" (p. 6). Sousa, Pimenta e Machado (2012), por sua vez, esclarecem que em Marília - SP, a devolutiva dos resultados se dá "após mais ou menos um mês da aplicação das provas" (p. 27). Em ambos municípios há um forte empenho para que as escolas tenham acesso aos resultados com a maior celeridade possível, mais precisamente cerca de um mês após a aplicação da avaliação. Assim sendo, a questão do rápido acesso aos resultados da avaliação aparece como uma necessidade das secretarias municipais de educação, que não escondem suas críticas à demora na devolutiva dos resultados da avaliação nacional e estadual.

\section{Quanto à forma de apresentação dos resultados}

No tratamento da temática em apreciação, a maioria das investigações expõe o formato sob o qual os resultados da avaliação têm sido apresentados. As variadas estratégias adotadas para publicação desses resultados constam na tabela 10.

Tabela 10 -

Forma de apresentação dos resultados.

\begin{tabular}{c|c|c}
\hline Formato de apresentação & Estudo & Total \\
\hline Relatórios & $\mathrm{B}, \mathrm{C}, \mathrm{D}, \mathrm{E}, \mathrm{I}, \mathrm{M}, \mathrm{Q}$ & 2 \\
\hline Gráficos & $\mathrm{I}, \mathrm{P}$ & 2 \\
\hline Boletins & $\mathrm{G}, \mathrm{I}$ & 1 \\
\hline Planilhas & $\mathrm{I}$ & 1 \\
\hline Tabelas & $\mathrm{P}$ & 1 \\
\hline Mapas de aproveitamento & $\mathrm{P}$ & 3 \\
\hline Não especificado & $\mathrm{H}, \mathrm{L}, \mathrm{R}$ & 5 \\
\hline Não menciona & $\mathrm{A}, \mathrm{F}, \mathrm{J}, \mathrm{O}, \mathrm{S}$ & \\
\hline
\end{tabular}

Os estudos, de um modo geral, além de indicar o formato - boletins, gráficos, planilhas, relatórios ou tabelas - escolhido pelas secretarias, detalham como foram estruturados esses resultados. No tocante a isso os estudiosos mencionam o esforço de 
consolidação dos resultados por Estado ${ }^{5}$, por município, por escola, por série/ano, por turma, por turma e descritor, por disciplina/área do conhecimento, por questão, por descritor, por conteúdo, por aluno, por acertos na turma e/ou por acertos em cada questão. Dentre essas opções, os dados foram mais frequentemente disponibilizados por turma e por aluno.

O fato é que quanto mais detalhado for e quanto mais rápido forem disponibilizados, maiores são as chances desses resultados serem utilizados em benefício da melhoria da aprendizagem dos alunos. No município pesquisado por Santos e Amorim Neto (2006), por exemplo,

o foco nos descritores básicos está fortalecendo o planejamento escolar e a elaboração de atividades diversificadas, uma vez que se tem relatório por escola/série/turma, contendo a média de desempenho dos alunos por descritores. Isto auxilia os professores no momento de planejar as aulas, pois podem atuar diretamente nos descritores de menor rendimento e avançar naqueles de bom desempenho. (p. 80)

Para uma efetiva apropriação desses resultados é essencial que esses dados sejam compreensíveis e que também apresentem informações qualitativas, só assim tais resultados podem se tornar um aliado do trabalho docente, pois viabilizarão 0 desenvolvimento da intervenção pedagógica.

\section{Quanto às limitações}

No processo analítico efetivado, determinados aspectos levantados por alguns dos estudos visitados foram reconhecidos como entraves associados às avaliações realizadas pelos municípios. Em síntese, a ausência de diálogo entre a secretaria municipal de educação e os professores em relação à avaliação (Castro; Siqueira, 2016), a restrição da avaliação municipal à função gerencialista (Aguiar, 2014), a data de aplicação da avaliação imprópria para o desenvolvimento da intervenção pedagógica (Aguiar, 2014), a realização da avaliação sob condições escassas (Alavarse; Bravo; Machado, 2013) e, por fim, a fragilidade técnica da avaliação municipal (Sousa, Pimenta E Machado, 2012) foram as limitações percebidas.

Do mesmo modo que foram identificados nas avaliações abordadas por esses estudos, é provável que alguns desses pontos críticos estejam presentes em outras propostas municipais de avaliação. Autores como Freitas et al. (2009), por exemplo, salientam que o estabelecimento de sistemas de avaliação no âmbito municipal esbarra nas condições das redes de ensino "desqualificadas pela política local que não privilegia a continuidade das ações e nem sempre a escolha dos melhores profissionais para ocupar cargos estratégicos" (p. 48).

Análises nessa direção, portanto, fazem-se necessárias, identificando eventuais limitações que venham a existir, e, sobretudo, servindo de alerta aos propositores dessas iniciativas, pois caso tais práticas sejam permeadas por falhas, elas poderão ocasionar mais danos do que ganhos às redes de ensino. Deficiência relativa ao rigor técnico da avaliação, pela sua gravidade e por ser alicerce sob o qual a avaliação se assenta, é, por sinal, motivo suficiente para implicar a desarticulação da iniciativa própria de avaliação.

${ }^{5}$ Especificamente no estudo de Marques; Ribeiro; Ciasca (2008).

Regae: Rev. Gest. Aval. Educ. 


\section{Considerações finais}

Com base nas descobertas efetuadas nota-se que, no âmbito dos municípios, a implantação de uma avaliação própria é entendida como algo imprescindível à gestão da educação, servindo a diversificadas finalidades. Grosso modo, ao mesmo tempo em que servem para nortear ações, a avaliação municipal e os seus resultados também servem para informar se as ações têm surtido efeito na aprendizagem dos estudantes.

Com efeito, tal implantação não acontece sem propósito, guardando vinculação com os sistemas de avaliação já existentes. A bem da verdade, as avaliações municipais se situam entre a crítica e a conformação aos sistemas de avaliação nacionais e estaduais.

As críticas a esses sistemas são tecidas pelas secretarias municipais de educação como uma forma de justificar a criação e a manutenção de suas iniciativas próprias de avaliação. Assim sendo, as secretarias passam a investir em avaliações próprias como uma forma de suprimir necessidades que os sistemas avaliativos em vigor não têm atendido, principalmente aquelas relativas ao conhecimento detalhado dos aspectos avaliados e ao rápido acesso aos resultados da avaliação.

$\mathrm{Na}$ medida em que pode se alinhar à crítica aos sistemas de avaliação de nível estadual e federal, a avaliação municipal também pode atuar como um mecanismo de afirmação desses sistemas, efetivando uma verdadeira inclinação às avaliações de maior escala. Por assim se revelar, em tal tendência coadunam-se experiências municipais de avaliação que se espelham nos sistemas de avaliação de cunho nacional e estadual, tomando-os como modelos a serem reproduzidos pela avaliação municipal. $O$ fato de as séries e as disciplinas mais frequentemente contempladas pelas avaliações municipais serem as mesmas já examinadas pelas avaliações externas de maior abrangência, se constitui em um considerável indício dessa inclinação.

Para além da busca de imprimir às avaliações municipais o protocolo adotado pelas avaliações tomadas como referência, determinadas avaliações concentradas nessa perspectiva são concebidas com vistas à preparação, ou ao treinamento, dos alunos para as demais avaliações externas. Nessa perspectiva, as avaliações externas de maior escala representam para as avaliações municipais, ao mesmo tempo, o ponto de partida e o ponto a que se pretende chegar. Visto sob esse ângulo, as avaliações municipais surgem a partir delas e para elas.

\section{Referências}

AGUIAR, Luzanilde Oliveira. Ampliando a funcionalidade pedagógica do Sistema de Avaliação da Educação Municipal de Juazeiro/BA - Saej. CONGRESSO NACIONAL DE AVALIAÇÃO EM EDUCAÇÃO, 3, 2014. Anais...Bauru: Unesp, 2014.

ALAVARSE, Ocimar M; BRAVO, Maria Helena; MACHADO, Cristiane. Avaliações externas e qualidade na educação básica: articulações e tendências. Est. Aval. Educ., São Paulo, v. 24, n. 54, 2013, p. 12-31.

BARRETO, Elba Siqueira de Sá; PINTO, Regina Pahim. Avaliação na educação básica 1990-1998. Brasília: MEC/Inep/Comped, 2001.

BAUER, Adriana. Avaliação em larga escala em municípios brasileiros: o que dizem os números? CONGRESSO NACIONAL DE AVALIAÇÃO EM EDUCAÇÃO, 3, 2014, Anais ... Bauru: Unesp, 2014. 
BAUER, Adriana; HORTA NETO, João Luiz; SOUSA, Sandra Zákia Lian. Avaliação e gestão educacional em municípios brasileiros: mapeamento e caracterização de iniciativas em curso. São Paulo: FCC/Inep, 216.

BAUER, Adriana; PIMENTA, Claudia Oliveira; HORTA NETO, João Luiz; SOUSA, Sandra Zákia Lian. Avaliação em larga escala em municípios brasileiros: o que dizem os números? Est. Aval. Educ., São Paulo, v. 26, n. 62, 2015, p. 326-352.

BREVES, Núbia do Socorro Pinto; MONTECONRADO, Glenda Martins; ROCHA, Santana Elvira Amaral da. Avaliação de desempenho do estudante na rede pública municipal de ensino de Manaus: estratégias para tomada de decisões. CONGRESSO NACIONAL DE AVALIAÇÃO EM EDUCAÇÃO, 4, 2016, Anais ... Bauru: Unesp, 2016.

CATALANI, Érica Maria Toledo; GONSALES, Fernando. Prova da Cidade 2009: uma experiência de avaliação em larga escala para diagnóstico da área de Matemática na cidade de São Paulo. CONGRESSO NACIONAL DE AVALIAÇÃO EM EDUCAÇÃO, 1, 2010, Bauru. Anais ... Bauru: Unesp, 2010, p. 1-19.

CARVALHO, Débora Aldyane Barbosa. O sistema de avaliação do desempenho escolar de Jaguaruana/CE - Sadej - para a prática pedagógica. Fortaleza: UFCE, 2013. 122f. Dissertação (mestrado em Educação). Faculdade de Educação, Universidade Federal do Ceará.

CASTRO, Arlene Moreno de; SIQUEIRA, Valéria Aparecida de Souza. Limites e potencialidades de iniciativas municipais de avaliação: um estudo de caso na RME/SP. CONGRESSO NACIONAL DE AVALIAÇÃO EM EDUCAÇÃO, 4, 2016. Anais ... Bauru: Unesp, 2016.

COSTA, Marly de Abreu; OLIVEIRA, Eloiza da Silva Gomes de. Avaliação do desempenho escolar de alunos da rede municipal de Angra dos Reis: subsídios para tomada de decisões. CONGRESSO INTERNACIONAL EM AVALIAÇÃO EDUCACIONAL, 3, 2006, Fortaleza. Anais ... Fortaleza, UFC, 2006.

COUTINHO, Magno Sales. Avaliação externa e currículo: possíveis impactos e implicações no processo de ensino aprendizagem. ENCONTRO NACIONAL DE DIDÁTICA E PRÁTICAS DE ENSINO, 16, 2012. Anais... Campinas: Unicamp, 2012, p. 18-28.

EVARISTO, Marcela Cristina; SANTOS, Ailton Carlos. Prova da Cidade 2009: uma experiência de avaliação em Língua Portuguesa na cidade de São Paulo. CONGRESSO NACIONAL DE AVALIAÇÃO EM EDUCAÇÃO, 1, 2010. Anais ... Bauru: Unesp, 2010.

FREITAS, Luiz Carlos de; SORDI, Mara Regina Lemes de; MALAVASI, Maria Marcia Sigrist; FREITAS, Helena Costa Lopes de. Avaliação de redes de ensino: a responsabilidade do poder público. In: FREITAS, Luiz Carlos de; SORDI, Mara Regina Lemes de; MALAVASI, Maria Marcia Sigrist; FREITAS, Helena Costa Lopes de. Avaliação educacional: caminhando pela contramão. Petrópolis, Rio de Janeiro: Vozes, 2009, p. 4768.

GIMENES, Nelson; SILVA, Vandré Gomes da; PRÍNCIPE, Lisandra Marisa; LOUZANO, Paula; MORICONI, Gabriela Miranda. Além da Prova Brasil: investimento em sistemas próprios de avaliação externa. Est. Aval. Educ., São Paulo, v. 24, n. 55, 2013, p. 12-32.

LIMA, Maria Deuzanira de; SANTOS, Geandra Cláudia Silva Santos; LIMA NETO, Francisco Feitosa; LIMA, Giuvanilda Gomes de Sousa. Avaliação da educação municipal de Tauá: Uma experiência em construção. CONGRESSO INTERNACIONAL EM AVALIAÇÃO EDUCACIONAL, 5, 2010. Anais ... Fortaleza: UFC, 2010, p. 2442-2449. 
MACHADO, Cristiane; ALAVARSE, Ocimar Munhoz; ARCAS, Paulo Henrique. Sistemas estaduais de avaliação: interfaces com qualidade e gestão da educação. Revista Brasileira de Política e Administração da Educação, v. 31, n. 3, 2015, p. 667-680.

MAROJA, Claudio. Prova São Paulo: um diálogo entre a avaliação em larga escala e externa e as orientações pedagógicas para a melhoria do ensino na cidade de São Paulo. CONGRESSO INTERNACIONAL EM AVALIAÇÃO EDUCACIONAL, 5, 2010. Anais ... Fortaleza: UFC, 2010, p. 2106-2113.

MARQUES, Cláudio de Albuquerque; RIBEIRO, Ana Paula de Medeiros; CIASCA, Maria Isabel Filgueiras Lima. Paic: o pioneirismo no processo de avaliação municipal com autonomia. Est. Aval. Educ., São Paulo: FCC, v. 19. n. 41, 2008, p. 433-448.

MARQUES, Maria do Carmo Pinheiro; BARROSO, Maria Cleide da Silva; SOUSA, Silvana Melo de. Política municipal de avaliação monitoramento e acompanhamento do rendimento escolar em Maracanaú. CONGRESSO INTERNACIONAL EM AVALIAÇÃO EDUCACIONAL, 5, 2010. Anais... Fortaleza: UFC, 2010, p. 2782-2789.

MUNIZ, Rita de Fátima; BRAGA, Adriana Eufrásio; MUNIZ, Sheila Maria. Avaliações em larga escala enquanto indutoras de ações: o caso do Saemjj. CONGRESSO INTERNACIONAL EM AVALIAÇÃO EDUCACIONAL, 6, 2015. Anais... Fortaleza: UFC, 2015.

MOREIRA, Rozemeiry dos Santos Marques. Avaliação externa como instrumento da gestão educacional: a adesão e os impasses de sua realização. Campinas: Unicamp, 2004. 136f. Dissertação (mestrado em Educação). Faculdade de Educação, Universidade Estadual de Campinas.

NUNES, Raquel Queiroz; SILVA, Gelcivânia Mota. Avaliação da aprendizagem escolar da rede pública municipal de ensino de Serrinha - Avapre. CONGRESSO NACIONAL DE AVALIAÇÃO EM EDUCAÇÃO, 4, 2016. Anais ... Bauru: Unesp, 2016.

OVANDO, Nataly Gomes. A avaliação na política educacional de municípios sul-matogrossenses. Dourados: UFGD, 2011. 66f. Dissertação (mestrado em Educação). Faculdade de Educação, Universidade Federal da Grande Dourados, 2011.

SANTOS, Maéve Melo dos; AMORIM NETO, Plínio José. Avaliação de ensinoaprendizagem no ensino fundamental: apresentação de metodologia desenvolvida pela secretaria municipal de ensino de Petrolina/PE. CONGRESSO INTERNACIONAL EM AVALIAÇÃO EDUCACIONAL, 3, 2006. Anais... Fortaleza: UFC, 2006.

SOUSA, Sandra Zakia; PIMENTA, Cláudia Oliveira; MACHADO, Cristiane. Avaliação e gestão municipal da educação. Est. Aval. Educ., São Paulo, v. 23, n. 53, 2012, p. 14-36.

Antônia Bruna da Silva é professora na Universidade do Estado do Rio Grande do Norte, vinculada ao Núcleo de Estudos em Educação.

Orcid: http://orcid.org/0000-0001-8609-2257.

Endereço: BR 405, km 3 - 59900-000 - Pau dos Ferros - RN - Brasil.

E-mail: brun.silwa@gmail.com. 
Maria Beatriz Fernandes é estudante no curso de Pedagogia da Universidade do Estado do Rio Grande do Norte, bolsista no Pibid/Capes.

Orcid: http://orcid.org/0000-0002-5532-8703.

Endereço: BR 405, km 3 - 59900-000 - Pau dos Ferros - RN - Brasil.

E-mail: beatrizfernandes98@hotmail.com.

Recebido em 8 de fevereiro de 2019.

Aceito em 16 de março de 2019

(c) (i) 